\title{
Prevalence of diabetes and impaired glucose tolerance in patients with schizophrenia
}

\author{
CHRIS BUSHE and RICHARD HOLT
}

\begin{abstract}
Background A number of studies have examined the prevalence of diabetes mellitus and impaired glucose tolerance in general populations and in those with schizophrenia and other forms of serious mental illness.
\end{abstract}

Aims To establish whether it is possible to describe accurately comparative rates of diabetes mellitus and impaired glucose tolerance in populations of people with schizophrenia and those without mental illness.

Method Review of current literature.

Results Research published in the preneuroleptic era suggested that people with severe mental illness were at increased risk of developing glycaemic abnormalities. Recent studies appear to confirm that the prevalence of diabetes and impaired glucose tolerance may be higher in people with schizophrenia than in the general population, and suggest that patients with schizophrenia have impaired glucose tolerance even before they begin treatment.

Conclusions Schizophrenia may be a significant and independent risk factor for both diabetes and impaired glucose tolerance. Current data preclude precise estimates of the prevalence of these conditions among people with schizophrenia.

Declaration of interest C.B. is an employee of Eli Lilly \& Co; R.H. has received educational grants and fees for lecturing and consultancy work from Eli Lilly \& Co.
The question of whether people with schizophrenia are at greater risk of developing type 2 diabetes than the general population has been puzzling researchers and clinicians since the early 1900s - long before the introduction of antipsychotic medications. As far back as 1897, the eminent Victorian psychiatrist Henry Maudsley (1835-1918) noted, 'Diabetes is a disease which often shows itself in families in which insanity prevails' (Maudsley, 1979). More than a century later, and after increasingly sophisticated research into the issue, Maudsley's intuitive observations about the association between severe psychiatric disorders and diabetes still appear to hold true.

Although early naturalistic approaches to the study of diabetes in patients with severe mental illness provided clues that an association did exist, it has been the more recent cross-sectional epidemiological studies assessing the prevalence of diabetes in populations of people with schizophrenia that have established beyond reasonable doubt that such people are at increased risk of developing diabetes, and that schizophrenia should now be considered to be an independent risk factor for the condition.

\section{METHOD}

Review of current literature.

\section{Confirming the association: research challenges}

Studies in the pre-neuroleptic era clearly suggested that schizophrenia might be a risk factor for the development of diabetes (Kooy, 1919; Lorenz, 1922; Henry \& Mangan, 1925; Freeman et al, 1944; Braceland et al, 1945; Freeman, 1946). However, many different criteria were used to define both schizophrenia and diabetes mellitus in these studies, and the 'pre-diabetic' state of impaired glucose tolerance has only relatively recently been defined (World Health
Organization, 1999). Today, standardisation of criteria for the diagnosis of diabetes, impaired glucose tolerance and schizophrenia make accurate and reproducible diagnoses possible, and allow epidemiological comparisons to be made across populations.

In order to establish definitively an association between schizophrenia and diabetes, it would be necessary for researchers to show that the prevalence of diabetes is higher among people with schizophrenia than in reference populations using modern research techniques and standardised disease definitions. Unfortunately, establishing the true prevalence of impaired glucose tolerance and/or diabetes in any population is fraught with difficulty. Diabetes has a gradual and insidious onset and is frequently asymptomatic, thereby explaining in part why up to half of all individuals with diabetes are thought to remain undiagnosed in the community (Harris et al, 1987; Mykkänen et al, 1990; Harris, 1993).

Prospective population-based studies in which there is universal screening for impaired glucose tolerance and diabetes using recommended blood glucose testing methods and established diagnostic criteria (World Health Organization, 1999) provide the best estimates of diabetes prevalence in the general population. Unfortunately, few such studies have ever been conducted, and because of the marked differences in diabetes rates between countries, social classes and age groups, it is difficult to extrapolate their estimates to other populations. Establishing the true prevalence of impaired glucose tolerance and diabetes in patients with schizophrenia is even more challenging. Difficulties with the identification and diagnosis of patients with diabetes are further compounded by inconsistencies in the diagnosis of schizophrenia. Some diabetes prevalence studies such as that by Cohen et al (2003) have evaluated heterogeneous populations that have included individuals with schizoaffective disorder, bipolar disorder and personality disorders, as well as those with schizophrenia, and although these studies provide much valuable information, they must be interpreted with care, since diabetes prevalence estimates do differ between these psychiatric conditions (Regenold et al, 2002).

Patients with schizophrenia are often reluctant to give consent to participate in epidemiological studies - especially ones that require blood samples to be taken (Cohen et al, 2003). This may result in 
prevalence estimates that are based on a self-selecting population and are therefore not necessarily an accurate reflection on the true picture. Even when patients consent to participate in diabetes prevalence studies, many investigators struggle to successfully complete oral glucose tolerance testing (Hägg et al, 1998), or even to obtain fasting blood glucose levels in their patients. This can lead to high study drop-out rates, or the use of less reliable indicators of glucose tolerance such as non-fasting blood glucose levels (Mukherjee et al, 1996; Cohen et al, 2003). Clearly, failure to perform fasting blood glucose tests could lead to an overestimate of diabetes prevalence.

Finally, in order to test the hypothesis that people with schizophrenia are at greater risk of developing diabetes than the general population, it is important that comparator populations are well matched for geographical region, age, gender, ethnicity and body mass index, and that as many other potential confounding factors are controlled for. Ideally, neither population should be taking medications that might alter the natural course of diabetes.

\section{RESULTS}

\section{Prevalence of diabetes in the general population}

Notwithstanding the methodological challenges outlined above, it is now clear that the prevalence of diabetes in the general population is high and increasing year by year (Mokdad et al, 2001). Studies in the USA have found rates of known diabetes in the general population of between $1.2 \%$ (for people aged 18-44 years) and $6.3 \%$ (for people aged 45-64 years) (Adams \& Marano, 1995), with the highest prevalence rates reported in African Americans, Native Americans and Mexican Americans (Adams \& Marano, 1995; Harris, 1998).

The Isle of Ely Diabetes Project in the UK, which was a prospective populationbased study, evaluated over a thousand individuals aged 40-65 years who were not known to have diabetes (Williams et al 1995). Individuals underwent a standard $75 \mathrm{~g}$ oral glucose tolerance test (OGTT) and were classified according to World Health Organization (WHO) criteria. This study found that $4.5 \%$ of participants had undiagnosed diabetes and $16.7 \%$ had impaired glucose tolerance (Williams et al, 1995). A similar large, population-based screening study in Australia (Dunstan et al, 2002) found an overall prevalence of diabetes of $7.4 \%$, and identified an additional $16.4 \%$ of the population with impaired glucose tolerance or impaired fasting blood glucose, according to WHO criteria.

Studies in Japan (Tabata et al, 1987) and Singapore (Singapore Ministry of Health, 1998) show prevalence rates of diabetes to be around $5 \%$ and $9 \%$, respectively, and confirm that twice as many people in the general population may have impaired glucose tolerance as have diabetes.

\section{Prevalence of diabetes in schizophrenia}

Studies assessing the prevalence of type 2 diabetes in people with schizophrenia broadly agree that the condition may be at least two to four times more prevalent than in the general population (Keskiner et al, 1973; Mukherjee et al, 1996; Dixon et al, 2000; Sernyak et al, 2002; Lindenmayer et al, 2003; Subramaniam et al, 2003). There is, however, significant variability in the reported prevalence rates from different studies. One major confounder when attempting to establish a true prevalence figure in schizophrenia and other populations is the number of people who have been actively screened. Citrome et al (2003) have recently reported that, in a retrospective epidemiological study evaluating the incidence of newly diagnosed diabetes mellitus in patients commencing antipsychotic treatment, some of the apparent differences in risk between individual agents could be attributed to the greater degree of glucose monitoring in patients receiving clozapine compared with those receiving other antipsychotics.

Another important factor that might influence prevalence figures reported in schizophrenia studies is the design of the study. Some studies require glucose screening (and possible exclusion for glycaemic abnormalities) prior to patient enrolment; others are cross-sectional in nature. In the latter case, all known confounders (such as population age, ethnicity and geography) must be accounted for. Even the year of the study and the type of schizophrenia diagnosed in the patients enrolled may affect prevalence estimates. In 2003, for example, improved awareness of the possibility of blood glucose abnormalities meant that more patients taking antipsychotic medications might have been screened for diabetes, which might account for the apparently higher prevalence rates being reported compared with 10 years ago.

Patients with treatment-resistant schizophrenia may be more likely to be screened for glucose abnormalities than other people with schizophrenia, and those that showed positive would probably be excluded from prevalence trials, a fact illustrated clearly by Lindenmayer et al (2003), who found a prevalence of diabetes mellitus of only $7 \%$ in their cohort of patients with treatmentresistant schizophrenia prior to study entry.

Mukherjee et al (1996) found an overall prevalence of known diabetes of $15.8 \%$ in a cohort of patients with schizophrenia admitted to a long-term care facility in Italy; diabetes was significantly more common in patients not receiving antipsychotics than in those who were. This overall prevalence rate is substantially higher than the known prevalence of diabetes in the general population of Italy, which is estimated to be 2-3\% (Verrillo et al, 1985; Bruno et al, 1992). The prevalence of known diabetes in Mukherjee's patient cohort increased with age, from $0 \%$ in individuals younger than 50 years, to $12.9 \%$ in the $50-59$ years age group, and to $18.9 \%$ in the $60-69$ years age group (Mukherjee et al, 1996). The prevalence of diabetes decreased to $16.7 \%$ in patients aged $70-74$ years and to $9.4 \%$ in those aged over 75 years, which has been interpreted by the investigators to indicate selective mortality due to coronary heart disease (Mukherjee et al, 1996).

Dixon et al (2000) used a large national database of patients receiving treatment for schizophrenia compiled by the Schizophrenia Patient Outcomes Research Team (PORT) in the USA to assess the prevalence and clinical correlates of known diabetes in this population. Much of the data were collected before the widespread use of novel antipsychotic medications. The lifetime prevalence of known diabetes reported for the people with schizophrenia in this study was $15 \%$, with increasing age, being female and being of African American or 'other' non-White racial origin increasing the likelihood of having diabetes (Dixon et al, 2000). The $15 \%$ prevalence of diabetes found in this study far exceeded rates typically reported in age-matched general populations, but this probably reflects the exhaustive interviewing techniques used in the study.

Sernyak et al (2002) reported an overall prevalence of diagnosed diabetes of $18 \%$ in a large sample of people with schizophrenia being treated as out-patients 
with typical and atypical antipsychotic drugs. Prevalence increased with age, with the highest prevalence $(>25 \%$ ) being seen in patients aged 60-69 years. Comparing the prevalence of known diabetes across age groups, no difference was found between patients who received an atypical antipsychotic and patients who received a typical antipsychotic (Sernyak et al, 2002).

Subramaniam et al (2003) conducted a chart review of 607 people with schizophrenia resident in a long-stay ward in Singapore. None of the patients had received atypical antipsychotic medication either in the past or at the time of the study. A prevalence of known diabetes of $4.9 \%$ was reported, and patients with this diagnosis were excluded from further study. Informed consent was then obtained to screen 194 of the remaining patients, and $16 \%$ of these were found to have diabetes, taking the total diabetes prevalence rate to around $21 \%$. Prevalence rates were found to be double those seen in the general population of Singapore in patients under the age of 60 years (Table 1), but lower than the general population in those aged 60 years and above. In this study, the highest rate of diabetes $(50 \%)$ was found in patients aged 50-59 years (Subramaniam et al, 2003). Gupta et al (2003) reported a prevalence rate of $17 \%$ for diabetes in a chart review of 208 patients with psychotic disorders who were receiving antipsychotic medication. Fasting blood glucose levels were used to establish the diagnosis of diabetes in this study (Gupta et al, 2003).

Subramaniam et al (2003) report the prevalence of impaired glucose tolerance in people with schizophrenia is less well defined, but screening studies suggest that

Table I Age-specific prevalence rates of diabetes mellitus in a group of people with schizophrenia compared with the general population in Singapore

\begin{tabular}{ccc} 
Age (years) & \multicolumn{2}{c}{ Prevalence of diabetes mellitus (\%) } \\
\cline { 2 - 3 } & $\begin{array}{c}\text { Schizophrenia } \\
\text { group }\end{array}$ & $\begin{array}{c}\text { General } \\
\text { population }\end{array}$ \\
\hline $18-29$ & n.a. & 0.8 \\
$30-39$ & 4.0 & 3.3 \\
$40-49$ & 17.3 & 9.6 \\
$50-59$ & 50.0 & 21.8 \\
$60-69$ & 23.7 & 32.4 \\
$70-79$ & 5.0 & n.a. \\
\hline
\end{tabular}

n.a., data not available.

From Subramaniam et al (2003). it may be almost twice as high as the prevalence of diabetes. In their screening study, the overall prevalence of diabetes was found to be $21 \%$ but that of impaired glucose tolerance was $31 \%$. The study has also highlighted the substantial problem of underrecognition of diabetes and impaired glucose tolerance in people with schizophrenia. Although $21 \%$ of patients were identified as having diabetes once they were actively screened, only $4.9 \%$ of patients had previously received a diagnosis (Subramaniam et al, 2003). Another study (Cohn et al, 2002) found that active screening for impaired glucose tolerance and diabetes in another cohort of 153 patients who had been taking antipsychotic monotherapy for at least 3 months found that $31 \%$ of individuals had either diabetes or impaired glucose tolerance, and that in the majority $(67 \%)$ the condition was previously unidentified (Cohn et al, 2002). Taylor also describes similar findings in 607 patients with schizophrenia at the Maudsley Hospital in London (Taylor et al, 2003; D. Taylor, personal communication, 2003). An apparent prevalence rate of glycaemic abnormalities (diabetes and impaired glucose tolerance) of $8.6 \%$ rose to $19.4 \%$ after prospective blood glucose testing.

Ryan and colleagues reported results from a landmark study involving young (mean age 33.6 years) patients with firstepisode schizophrenia. All patients were drug-naive, allowing a separation of the effects of the disease from the potential confounding effects of any antipsychotic medication. Despite being young and never having been exposed to neuroleptics, more than $15 \%$ of the individuals in this study had already developed impaired fasting glucose tolerance, compared with none of the matched healthy control groups (Ryan et al, 2003). This provides some of the clearest evidence that schizophrenia itself may be an independent risk factor for impaired glucose tolerance, which is a known risk factor for the development of type 2 diabetes (Alberti, 1996). Ryan et al (2003) contrast the prevalence of impaired glucose tolerance found in their study with that recently reported from a general population study in three regions of France (Gourdy $e t$ $a l, 2001)$. The mean age of the Ryan cohort was 33 years, and the age-adjusted (35-64 years) prevalence of impaired glucose tolerance found in the French study was $11.8 \%$ in men and $5.2 \%$ in women. The prevalence of impaired glucose tolerance in the population with schizophrenia was therefore found to be twice that in a general population (Ryan et al, 2003).

\section{Prevalence of diabetes in other psychiatric conditions}

Schizophrenia is not the only severe mental illness associated with an apparent increase in the risk of diabetes. Regenold et al (2002) assessed the medical records of 243 in-patients, aged $50-74$ years, with a diagnosis of major depression, bipolar I disorder, schizoaffective disorder, schizophrenia or dementia, and compared their rates of diabetes with rates in the US general population matched for age, gender and race. The prevalence of diabetes was found to be significantly higher than national norms in patients with bipolar I disorder $(26 \%)$ and schizoaffective disorder $(50 \%)$, and was independent of psychotropic drug use (Regenold et al, 2002). This study, and the work of others (Cassidy et al, 1999), indicate that the prevalence of diabetes in patients with bipolar disorder may be two to three times higher than that found in the general population.

\section{Schizophrenia: an independent risk factor for diabetes?}

The interaction between diabetes and schizophrenia, although relatively well established, is far from simple. The mechanisms behind the interaction are likely to be multifactorial, and to include genetic and environmental factors, as well as the possible effects of antipsychotic medications.

\section{Genetic factors}

Genetic factors appear to have a key role in the association between schizophrenia and diabetes, since it has been reported that up to $50 \%$ of individuals with schizophrenia have a family history of type 2 diabetes, compared with just $4.6 \%$ of healthy adult controls (Dynes, 1969; Mukherjee et al 1989; Cheta et al, 1990; Lamberti et al, 2003; Shiloah et al, 2003). In one of the largest chart reviews ever conducted of schizophrenia, Lamberti et al (2003) found a family history of type 2 diabetes in $17 \%$ of the total cohort of 436 patients. Importantly, in the cohort of patients who had a positive family history of diabetes, the prevalence of diabetes mellitus was $33 \%$. In those with a negative family history of diabetes, its prevalence 
was just $10 \%$. These data-sets suggest that genetic factors may explain to some extent the higher diabetes prevalence figures found in patients with schizophrenia compared with the general population.

\section{Environmental factors}

Many people with schizophrenia exhibit poor health behaviours that may also contribute to them developing diabetes; these include having less healthy diets (generally high in fat and low in fibre), exercising less and smoking more than normal controls (Brown et al, 1999). Poverty, unstable living conditions and lower than expected educational attainment are all associated with schizophrenia, and increase the risk of obesity and other adverse medical sequelae (Dixon et al, 2000). Additional factors thought to predispose individuals with schizophrenia to developing diabetes include ethnicity, a history of glucose dysregulation, and pre-existing hypertension (Lindenmayer et al, 2001).

The role of antipsychotic medications in the development of diabetes is far from well defined. Evidence from studies published before the introduction of neuroleptic drugs showed a strong association between severe mental illness and abnormal glucose metabolism (Kooy, 1919; Lorenz, 1922; Henry \& Mangan, 1925; Freeman et al, 1944; Freeman, 1946; Braceland et al, 1945), and although this evidence must be interpreted with care because of the definitions used in the studies, it suggests that schizophrenia itself might be an independent risk factor for the development of diabetes. Reports of impaired glucose tolerance in young, drug-naïve individuals with first-episode schizophrenia (Ryan et al, 2003) add further weight to the argument that people with schizophrenia may be naturally predisposed to developing diabetes, and should therefore be considered to be a high-risk group.

\section{DISCUSSION}

People with schizophrenia and other serious psychiatric conditions appear to be at significant risk of developing impaired glucose tolerance and diabetes, regardless of whether they are receiving antipsychotic medication. Many of these individuals at risk are currently unrecognised. Cardiovascular risk factors such as diabetes, hypertension and dyslipidaemia commonly coexist (Mukherjee et al, 1996), so it seems

\section{CLINICAL IMPLICATIONS}

Individuals with schizophrenia and other severe psychiatric conditions are at greater risk of developing diabetes mellitus and impaired glucose tolerance than the general population.

Approximately $15 \%$ of patients with schizophrenia may have diabetes, and a similar percentage may have impaired glucose tolerance. Increasing age is associated with increased risk.

Routine screening and the development of pragmatic pathways for diabetes risk management in all patients with schizophrenia are recommended.

\section{LIMITATIONS}

The true prevalence of diabetes and impaired glucose tolerance in all populations may still be grossly underestimated because of the silent nature of the disease.

- The association between schizophrenia and diabetes has been established on the basis primarily of early case reports and evidence from retrospective studies.

- Only large-scale, prospective, longitudinal studies in which all potential confounders are controlled for will establish the precise nature of the association.

CHRIS BUSHE, MB BS, Eli Lilly \& Co. Ltd, Basingstoke; RICHARD HOLT, MA, MB, BChir, PhD, MRCP (UK), Endocrinology and Metabolism Sub-division, Fetal Origins of Adult Disease Division, University of Southampton, UK

Correspondence: Dr Chris Bushe, Eli Lilly \& Co. Ltd, Lilly House, Priestley Road, Basingstoke RG24 9NL, UK. Tel: (0)I256 77597I; fax: (0)I256 775534; e-mail: bushe_chris@lilly.com

likely that these 'missing millions' of people with impaired glucose tolerance and diabetes may also have comorbidities that further increase their risk of cardiovascular disease and premature death (Subramaniam et al, 2003).

Natural causes are responsible for twothirds of the excess mortality associated with schizophrenia (Brown et al, 2000). In one record-linkage study, ischaemic heart disease was found to be the most common cause of death in both men and women with schizophrenia (Herman et al, 1983).

Age, ethnic origin and geographical location all appear to alter the prevalence of diabetes mellitus and impaired glucose tolerance. Early identification of these conditions in high-risk groups is now a priority in many countries. Diabetes UK currently recommends routine screening for diabetes in White people over the age of 40 years and in people from Black, Asian and minority ethnic groups aged over 25 years if they have a first-degree family history of diabetes, and/or are overweight and have a sedentary lifestyle, and/or have cardiovascular disease (Diabetes UK, 2002).

The strength of the association between schizophrenia and diabetes is such that timely screening and effective management of diabetes risk factors in all our patients is now recommended. Early detection of impaired glucose metabolism and effective education about healthy living should help to reduce the risk of patients developing diabetes and its complications, and may ultimately help to improve long-term outcomes.

\section{REFERENCES}

Adams, P. F. \& Marano, M. A. (1995) Current Estimates from the National Health Interview Survey, 1994. Vital and Health Statistics Series 10, Number 193. DHHS Publication PHS 96-152I. Hyattsville, MD: National Center for Health Statistics. 
Alberti, K. G. (1996) The clinical implications of impaired glucose tolerance. Diabetes Medicine, I3, 927-937.

Braceland, F. J., Meduna, L. J. \& Vaichulis, J. A. (1945) Delayed action of insulin in schizophrenia. American Journal of Psychiatry, 102, 108-110.

Brown, S., Birtwistle, J., Roe, L., et al (1999) The unhealthy lifestyle of people with schizophrenia. Psychological Medicine, 29, 697-701.

Brown, S., Barraclough, B. \& Inskip, H (2000)

Causes of the excess mortality of schizophrenia. British Journal of Psychiatry, 177, 212-217.

Bruno, G., Barbero, G., Vuolo, A., et al (1992) A population-based prevalence survey of known diabetes mellitus in Northern Italy based upon multiple independent sources of ascertainment. Diabetologia, 35 85I-856.

Cassidy, F., Ahearn, E. \& Carroll, B. J. (1999) Elevated frequency of diabetes mellitus in hospitalized manicdepressive patients. American Journal of Psychiatry, 156, 1417-1420.

Cheta, D., Dumitrescu, C., Georgescu, M., et al

(1990) A study on the types of diabetes mellitus in first degree relatives of diabetic patients. Diabetes and Metabolism, 16, II-15.

Citrome, L., Jaffe, A., Levine, J., et al (2003) Antipsychotic medication treatment and new prescriptions for insulin and oral hypoglycaemics. European Neuropsychopharmacology, 13 (suppl. 4), S306.

Cohen, D., Puite, B., Dekker, J., et al (2003) Diabetes mellitus in 93 chronic schizophrenic inpatients. European Journal of Psychiatry, 17, 38-47.

Cohn, T., Wolever, T., Zipursky, R., et al (2002) Screening for diabetes and impaired glucose tolerance in patients on antipsychotic medication. International Journal of Neuropsychopharmacology, 5 (suppl. I), SI68.

Diabetes UK (2002) Position Statement. Early Identification of People with Type 2 Diabetes. London: Diabetes UK. http://www. diabetes.org.uk/infocentre/ state/downloads/earlyid.doc

Dixon, L., Weiden, P., Delahanty, J., et al (2000) Prevalence and correlates of diabetes in national schizophrenia samples. Schizophrenia Bulletin, 26 903-912.

Dunstan, D. W., Zimmet, P. Z., Welborn, T. A., et al (2002) The rising prevalence of diabetes and impaired glucose tolerance: the Australian Diabetes, Obesity and Lifestyle Study. Diabetes Care, 25, 829-834.

Dynes, J. B. (1969) Diabetes in schizophrenia and diabetes in nonpsychotic medical patients. Diseases of the Nervous System, 30, 34I-344.

Freeman, H. (1946) Resistance to insulin in mentally disturbed soldiers. Archives in Neurology and Psychiatry, 56, 74-78.

Freeman, H., Rodnick, E. H., Shakow, D., et al (1944)

The carbohydrate tolerance of mentally disturbed soldiers. Psychosomatic Medicine, 6, 311-317.
Gourdy, P., Ruidavets, J. B., Ferrieres, J., et al (200I) Prevalence of type 2 diabetes and impaired fasting glucose in the middle-aged population of three French regions - the MONICA study. Diabetes and Metabolism 27, 347-358.

Gupta, S., Steinmeyer, C., Frank, B., et al (2003) Hyperglycemia and hypertriglyceridemia in real world patients on antipsychotic therapy. American Journal of Therapeutics, 10, 348-355.

Hägg, S., Joelsson, L., Mjorndal, T., et al (1998) Prevalence of diabetes and impaired glucose tolerance in patients with clozapine compared with patients treated with conventional depot neuroleptic medications. Journa of Clinical Psychiatry, 59, 294-299.

Harris, M. I. (1993) Undiagnosed NIDDM: clinical and public health issues. Diabetes Care, 16, 642-652.

Harris, M. I. (1998) Diabetes in America: epidemiology and scope of the problem. Diabetes Care, 2l (suppl. 3), ClI-14.

Harris, M. I., Hadden, W. C., Knowler, W. C., et al (1987) Prevalence of diabetes and impaired glucose tolerance and plasma glucose levels in US population aged 20-74 yr. Diabetes, 36, 523-534.

Henry, G. W. \& Mangan, E. (1925) Blood in personality disorders: biochemical studies. Archives of Neurology and General Psychiatry, 13, 743-749.

Herman, H. E., Baldwin, J. A. \& Christie, D. (1983) A record-linkage study of mortality and general hospital discharge in patients diagnosed as schizophrenic. Psychological Medicine, 13, 581-593.

Keskiner, A., El Toumi, A. \& Bousquet, T. (1973)

Psychotropic drugs, diabetes and chronic mental patients. Psychosomatics, 14, 176-181.

Kooy, F. H. (1919) Hyperglycemia in mental disorders. Brain, 42, 214-288

Lamberti, J., Crilly, J., Maharaj, K., et al (2003) Prevalence of adult-onset diabetes among outpatients receiving antipsychotic drugs. Schizophrenia Research, 60 (suppl.), S360.

Lindenmayer, J.-P., Nathan, A. M. \& Smith, R. C. (200I) Hyperglycaemia associated with the use of atypical antipsychotics. Journal of Clinical Psychiatry, 62, 30-38.

Lindenmayer, J.-P., Czobor, P., Volavka, J., et al (2003) Changes in glucose and cholesterol levels in patients with schizophrenia treated with typical or atypical antipsychotics. American Journal of Psychiatry, $\mathbf{1 6 0}$ 290-296.

Lorenz, W. F. (1922) Sugar tolerance in dementia praecox and other mental disorders. Archives of Neurology and Psychiatry, 8, 184-196.

Maudsley, H. (1979) The Pathology of Mind (3rd edn) London: Macmillan.

Mokdad, A. H., Ford, E. S., Bowman, B. A., et al (200I) The continuing increase of diabetes in the US. Diabetes Care, 24, 412 .
Mukherjee, S., Schnur, D. B. \& Reddy, R. (1989) Family history of type 2 diabetes in schizophrenic patients. Lancet, i, 495.

Mukherjee, S., Decina, P., Bocola, V., et al (1996) Diabetes mellitus in schizophrenic patients. Comprehensive Psychiatry, 37, 68-73.

Mykkänen, L., Laasko, M., Uusitupa, M., et al (1990) Prevalence of diabetes and impaired glucose tolerance in elderly subjects and their association with obesity and family history of diabetes. Diabetes Care, 13, 1099-II05.

Regenold, W. T., Thapar, R. K., Marano, C., et al (2002) Increased prevalence of type 2 diabetes mellitus among psychiatric inpatients with bipolar I affective and schizoaffective disorders independent of psychotropic drug use. Journal of Affective Disorders, 73, 301-302.

Ryan, M. C. M., Collins, P., Thakore, J. H. (2003) Impaired fasting glucose tolerance in first-episode, drugnaïve patients with schizophrenia. American Journal of Psychiatry, 160, 284-289.

Sernyak, M. J., Leslie, D. L., Alarcon, R. D., et al (2002) Association of diabetes mellitus with use of atypical neuroleptics in the treatment of schizophrenia. American Journal of Psychiatry, 159, 56I-566.

Shiloah, E., Witz, S., Abramovich, Y., et al (2003) Effect of psychotic stress in non-diabetic subjects on beta-cell function and insulin sensitivity. Diabetes Care, 26, 1462-1467.

Singapore Ministry of Health (1998) National Health Survey Singapore, pp. 5-9. Singapore: Epidemiology and Disease Control Department, Ministry of Health.

Subramaniam, M., Chong, S.-A. \& Pek, E. (2003) Diabetes mellitus and impaired glucose tolerance in patients with schizophrenia. Canadian Journal of Psychiatry, 48, 345-347.

Tabata, H., Kikuoka, M., Kikuoka, H., et al (1987) Characteristics of diabetes mellitus in schizophrenic patients. Journal of the Medical Association of Thailand, 70, 90-93.

Taylor, D., Young, C., Mahomed, R., et al (2003) A prevalence study of impaired glucose tolerance and diabetes mellitus in hospitalised patients with schizophrenia. European Neuropsychopharmacology, 13 (suppl. 4), S323.

Verrillo, A., De Teresa, A., La Rocca, S., et al (1985) Prevalence of diabetes mellitus and impaired glucose tolerance in a rural area of Italy. Diabetes Research, 2. 30I-306.

Williams, D. R., Wareham, N. J., Brown, D. C., et al (1995) Undiagnosed glucose intolerance in the community: the Isle of Ely Diabetes Project. Diabetic Medicine, 12, 30-35.

World Health Organization (1999) Report of a WHO Consultation: Definition, Diagnosis and Classification of Diabetes Mellitus and its Complications. Part I: Diagnosis and Classification of Diabetes Mellitus. WHO/NCD/ NCS/99.2. Geneva: WHO. 